\title{
PATIENTS WITH INFLAMMATORY MYOPATHIES OVERLAPPING WITH SYSTEMIC SCLEROSIS: A BRAZILIAN-JAPANESE BICENTRIC DESCRIPTIVE STUDY
}

Jucier Gonçalves Júnior ${ }^{1, *}$, Naoki Mugii ${ }^{2}$, Pleiades Tiharu Inaoka², Renata Miossi ${ }^{1}$, Fernando Henrique Carlos de Souza ${ }^{1}$, João Calvino Soares de Oliveira1, Fujiko Someya², Yasuhito Hamaguchi², Takashi Matsushita², Percival Degrava Sampaio-Barros ${ }^{1}$, Samuel Katsuyuki Shinjo ${ }^{1}$

1.Universidade de São Paulo, São Paulo (SP), Brazil; 2.Kanazawa University Hospital, Kanazawa (Ishikawa), Japan.

*Corresponding author: juciergjunior@hotmail.com

\section{BACKGROUND}

The literature contains few studies on inflammatory myopathies and systemic sclerosis overlap syndromes (MyoSScOS), and most of these are limited to reports or case series. Thus, we aim to analyze the demographic, clinical, and laboratory characteristics and follow-up of a representative sample of patients with MyoSScOS.

\section{METHODS}

A descriptive study was conducted with 45 patients with MyoSScOS from two tertiary centers (Brazil and Japan) using data from the period 2000-2020.

\section{RESULTS}

The patients, who were predominantly female, had a current average age of 50 years, and a median follow-up time of 98 months. Myo or SSc onset was earlier in Brazilian patients. Muscle impairment started simultaneously with the diagnosis of systemic sclerosis in $57.8 \%$ of cases. Muscle involvement occurred before the onset of systemic sclerosis in $35.5 \%$ of cases, and after in $6.7 \%$. Polymyositis was observed in $55.6 \%$ of cases, followed by dermatomyositis in $24.4 \%$ and antisynthetase syndrome in $20.0 \%$. Concerning systemic sclerosis, the diffuse and limited forms occurred in $64.4 \%$ and $35.6 \%$ of the cases, respectively. Comparing the subgroups, Brazilian patients had a higher frequency of dysphagia and digital ulcers, whereas Japanese patients had higher modified Rodnan skin score and prevalence of positive anticentromere antibodies. The current disease status and morbimortality were similar in both groups.

\section{CONCLUSION}

In the present study, MyoSSCOS affected middle-aged women, and its manifestation spectrum varies according to geographic distribution.

\section{KEYWORDS}

Systemic sclerosis, Dermatomyositis, Inflammatory myopathies, Myositis, Polymyositis. 